\title{
DÉPENDANCE ÉNERGÉTIQUE DES MATÉRIAUX PLASTIQUES COMME DÉTECTEURS SOLIDES DE TRACES POUR LES NEUTRONS RAPIDES
}

\author{
F. SPURNY, J. LOCHMANOVA, K. TUREK*
}

(Manuscrit reçu le 5 décembre 1974)

\begin{abstract}
RÉSUMÉ
Nous avons étudié la détection des neutrons rapides au moyen des réactions neutroniques sur les noyaux constituant les matériaux plastiques utilisables comme détecteurs solides de traces.

Nous avons calculé le nombre de particules chargées lourdes $(Z>2)$ dont les traces peuvent être développées et qui sont produites lors des réactions neutroniques dans des matériaux tels que le polycarbonate et les nitrate, triacétate et acétobutyrate de cellulose. Nous avons adopté plusieurs critères de développement de la trace d'une particule.

Nous avons étudié l'influence des conditions expérimentales choisies et nous avons montré que celles-ci sont très importantes. En particulier nous avons observé que l'attaque chimique par certaines solutions donne des sensibilités nettement supérieures à celles obtenues à l'aide de la soude en solution aqueuse. La comparaison des valeurs expérimentales avec le calcul théorique a montré que les traces des noyaux de recul ainsi que celles des particules alpha créées sont décelables si leur énergie dépasse environ $0,2 \mathrm{MeV}$.

Quant à l'application dosimétrique, les détecteurs étudiés devraient avoir une dépendance énergétique assez faible dans la région comprise entre $1,3 \mathrm{et} 14 \mathrm{MeV}$. La sensibilité dans cette région est, dans nos conditions expérimentales, de l'ordre de $10^{4}$ trace $\mathrm{cm}^{-2} \cdot \mathrm{rad}^{-1}$, lorsque le développement correspond à une attaque de la surface du matériau de $20 \mu \mathrm{m}$.
\end{abstract}

\section{ABSTRACT}

The detection of fast neutrons by the registration of recoil nuclei in plastic solid state track detectors was studied.

The number of heavy charged particles $(Z>2)$ formed by the interactions of fast neutrons in plastics (polycarbonate, cellulose triacetate, acetobutyrate and nitrate) where their tracks are etchable were calculated for several assumptions as regard the etchability of tracks.

(*) Laboratoire de Dosimétrie Radiologique, Académie des Sciences de Tchécoslovaquie, Na Truhlarce 39/29, 18086 Praha 8, Tchécoslovaquie. 
The influence of etching conditions was studied ; it was very important, particularly, in our experimental conditions, we observed that the sensitivity was lower for $\mathrm{NaOH}$ solutions than for some other solutions.

The comparison of the experimental and theoretical results showed that the tracks of alpha particles as well as recoil nuclei were etchable if their energy was higher than about $0.2 \mathrm{MeV}$.

As for dosimetric applications, the detectors studied should be energetically independent for neutrons of energy between 1.3 and $14 \mathrm{MeV}$; in our experimental conditions the sensitivity in the region was $10^{4}$ track. $\mathrm{cm}^{-2} \cdot \mathrm{rad}^{-1}$ for a surface removal $h=20 \mu \mathrm{m}$.

\section{INTRODUCTION}

Une des applications les plus intéressantes des détecteurs solides de traces est la dosimétrie des neutrons [1,2]. Des ensembles constitués par ces détecteurs et des matériaux fissiles sont même utilisés pour la dosimétrie de routine [3]

On sait que les matériaux plastiques eux-mêmes peuvent détecter les neutrons par suite des réactions neutroniques avec les noyaux constituant le matériau. Les auteurs confirment que, dans ce cas, la sensibilité est comparable et même supérieure à celle des ensembles avec convertisseurs fissiles [4-11]. En même temps, cette méthode présente des avantages tels que la simplicité, le prix modique, etc. C'est pourquoi il nous a paru intéressant d'effectuer une étude plus complète de cette méthode. La première partie de ce travail est une analyse théorique de la dépendance énergétique du nombre des traces décelées pour les matériaux tels que les polycarbonates et les composés cellulosiques. Dans la seconde partie, on donne les résultats expérimentaux obtenus auprès des sources Am-F, Am-Be et avec des neutrons de $14 \mathrm{MeV}$. Ces résultats sont comparés avec le calcul théorique. En conclusion, on indique les possibilités de la méthode pour la dosimétrie des neutrons.

\section{ANALYSE THÉORIQUE}

Soit $N_{j}$ le nombre de noyaux du type $j$ dans $1 \mathrm{~cm}^{3}$ d'un matériau plastique. Le nombre $C\left(E_{n}\right)$ de particules chargées lourdes $(Z>2)$ produites par un neutron d'énergie $E_{n}$ dans la couche $\Delta X$ du matériau et dont les traces sont développables est donné par l'équation :

$$
C\left(E_{n}\right)=\sum_{i} \sum_{j} \eta_{i j}\left(E_{n}\right) \sigma_{i j}\left(E_{n}\right) N_{j} X
$$

où $\eta_{i j}\left(E_{n}\right)$ est un facteur de correction qui exprime quelle fraction des particules lourdes créées lors d'une réaction du type $i$ avec le noyau $j$ forme des traces développables.

$\sigma_{i j}\left(E_{n}\right)$ est la section efficace d'une réaction du type $i$ avec le noyau $j$. Les réactions considérées dans ce calcul sont présentées dans le tableau I, 
TABLEAU I

REACTIONS UTILISEES POUR LE CALCUL DES C( $\left.E_{n}\right)$

\begin{tabular}{|c|c|c|c|c|c|}
\hline \multirow{2}{*}{\multicolumn{2}{|c|}{ Réaction }} & \multirow[t]{2}{*}{ Particule } & \multicolumn{3}{|c|}{$\begin{array}{l}\text { Energie minimale des neutrons } \\
\text { pour que la particule soit enre- } \\
\text { gistrable d'après le groupe de } \\
\text { restrictions energétiques ( }(\mathrm{MeV}) \text {. }\end{array}$} \\
\hline & & & I & II & III \\
\hline${ }^{12} \mathrm{C}(\mathrm{n}, \mathrm{n}){ }^{12} \mathrm{C}$ & Diffusion élastique & $\mathrm{C}$ & 0,70 & 2,11 & 3,52 \\
\hline${ }^{12} \mathrm{C}\left(\mathrm{n}, \mathrm{n}^{\prime}\right){ }^{12} \mathrm{C}^{*}$ & $\mathrm{E}^{*}=4,43 \mathrm{MeV}$ & $\mathrm{C}$ & 7,00 & 12,30 & - \\
\hline${ }^{12} \mathrm{C}\left(\mathrm{n}, \mathrm{n}^{\prime}\right){ }^{12} \mathrm{C}^{*}$ & $\mathrm{E}_{\gamma}=6,8 \mathrm{MeV}$ & $\mathrm{C}$ & 11,10 & - & - \\
\hline \multirow[t]{2}{*}{${ }^{12} \mathrm{C}(\mathrm{n}, \alpha){ }^{9} \mathrm{Be}$} & $Q^{\prime}=-5,71 \mathrm{MeV}$ & $\alpha$ & 7,70 & 7,70 & 7,70 \\
\hline & & $\mathrm{Be}$ & 7,70 & 7,70 & 8,90 \\
\hline \multirow[t]{2}{*}{${ }^{12} \mathrm{C}(\mathrm{n}, \alpha){ }^{9} \mathrm{Be}^{*}$} & $\mathrm{E}^{*}=1,75 \mathrm{MeV}$ & $\alpha$ & 9,50 & 9,50 & 9,50 \\
\hline & & $\mathrm{Be}$ & 9,50 & 9,50 & - \\
\hline \multirow{2}{*}{$\begin{array}{l}{ }^{12} \mathrm{C}(\mathrm{n}, \mathrm{n}) 3 \alpha \\
{ }^{12} \mathrm{C}(\mathrm{n}, \alpha){ }^{9} \mathrm{Be}\end{array}$} & & $\alpha$ & 11,70 & 11,70 & 12,70 \\
\hline & $2 \alpha+\mathrm{n}$ & $\alpha$ & 10,30 & 10,30 & 10,30 \\
\hline \multirow{9}{*}{ 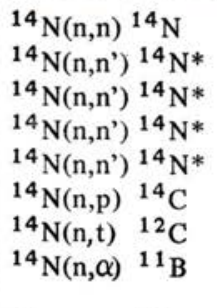 } & Diffusion élastique & $\mathrm{N}$ & 0,80 & 2,41 & 4,02 \\
\hline & $\mathrm{E}_{\gamma}=1,68 \mathrm{MeV}$ & $\mathrm{N}$ & 5,60 & 10,70 & - \\
\hline & $\mathrm{E}_{\gamma}=2,31 \mathrm{MeV}$ & $\mathrm{N}$ & 6,30 & 11,30 & - \\
\hline & $\mathrm{E}_{\gamma}^{\prime}=5,1 \quad \mathrm{MeV}$ & $\mathrm{N}$ & 8,10 & - & - \\
\hline & $\mathrm{E}_{\gamma} \sim 10 \mathrm{MeV}$ & $\mathrm{N}$ & 12,90 & - & - \\
\hline & $\mathrm{Q}^{\prime}=-0,63 \mathrm{MeV}$ & $\mathrm{C}$ & 2,37 & 8,37 & - \\
\hline & $\mathrm{Q}=-4,0 \mathrm{MeV}$ & $\mathrm{C}$ & 5,50 & 7,00 & 9,00 \\
\hline & $\mathrm{Q}=-0,16 \mathrm{MeV}$ & $\alpha$ & 1,30 & 1,30 & 1,30 \\
\hline & & B & 1,30 & 2,40 & 3,90 \\
\hline \multirow[t]{2}{*}{${ }^{14} \mathrm{~N}(\mathrm{n}, \alpha){ }^{11} \mathrm{~B}^{*}$} & $\mathrm{E}_{\gamma}=2,14 \mathrm{MeV}$ & $\alpha$ & 4,10 & 4,10 & 4,10 \\
\hline & & B & 4,10 & 4,50 & 7,00 \\
\hline \multirow[t]{2}{*}{${ }^{14} \mathrm{~N}(\mathrm{n}, \alpha){ }^{11} \mathrm{~B}^{*}$} & $\mathrm{E}_{\gamma}=4,46 \mathrm{MeV}$ & $\alpha$ & 6,10 & 6,10 & 6,10 \\
\hline & & B & 6,10 & 6,80 & 8,30 \\
\hline \multirow[t]{2}{*}{${ }^{14} \mathrm{~N}(\mathrm{n}, \alpha){ }^{11} \mathrm{~B}$} & $\mathrm{E}_{\boldsymbol{\gamma}}=5,0 \mathrm{MeV}$ & $\alpha$ & 7,10 & 7,10 & 7,10 \\
\hline & & B & 7,10 & 7,50 & 10,00 \\
\hline${ }^{16} \mathrm{O}(\mathrm{n}, \mathrm{n}){ }^{16} \mathrm{O}$ & Diffusion élastique & $\mathrm{O}$ & 0,90 & 2,71 & 4,52 \\
\hline${ }^{16} \mathrm{O}\left(\mathrm{n}, \mathrm{n}^{\prime}\right){ }^{16} \mathrm{O}^{*}$ & $\mathrm{E}_{\gamma}=6,1 \mathrm{MeV}$ & $\mathrm{O}$ & 9,50 & - & - \\
\hline${ }^{16} \mathrm{O}\left(\mathrm{n}, \mathrm{n}^{\prime}\right){ }^{16} \mathrm{O}^{*}$ & $\mathrm{E}_{\boldsymbol{\gamma}}=7,0 \mathrm{MeV}$ & $\mathrm{O}$ & 10,50 & - & - \\
\hline${ }^{16} \mathrm{O}\left(\mathrm{n}, \mathrm{n}^{\prime}\right)^{16} \mathrm{O}^{*}$ & $\mathrm{E}_{\gamma}=3,8 \mathrm{MeV}$ & O & 10,70 & 13,50 & - \\
\hline${ }^{16} \mathrm{O}\left(\mathrm{n}, \mathrm{n}^{\prime}\right)^{16} \mathrm{O}^{*}$ & $\mathrm{E}_{\gamma}=4,8 \mathrm{MeV}$ & $\mathrm{O}$ & 12,70 & - & - \\
\hline${ }^{16} \mathrm{O}(\mathrm{n}, \mathrm{p}){ }^{16} \mathrm{~N}$ & $Q^{\prime}=-9,63 \mathrm{MeV}$ & $\mathrm{N}$ & 13,00 & - & - \\
\hline \multirow{2}{*}{${ }^{16} \mathrm{O}(\mathrm{n}, \alpha){ }^{13} \mathrm{C}$} & $\mathrm{Q}=-2,21 \mathrm{MeV}$ & $\alpha$ & 3,80 & 3,80 & 3,80 \\
\hline & & C & 3,80 & 4,70 & 6,40 \\
\hline \multirow[t]{2}{*}{${ }^{16} \mathrm{O}(\mathrm{n}, \alpha){ }^{13} \mathrm{C}^{*}$} & $\mathrm{E}_{\boldsymbol{\gamma}}=3,1 \mathrm{MeV}$ & $\alpha$ & 7,60 & 7,60 & 7,60 \\
\hline & & C & 7,60 & 8,00 & 9,70 \\
\hline \multirow{2}{*}{${ }^{16} \mathrm{O}(\mathrm{n}, \alpha){ }^{13} \mathrm{C}^{*}$} & $\mathrm{E} \gamma \sim 3,8 \quad \mathrm{MeV}$ & $\alpha$ & 8,20 & 8,20 & 8,20 \\
\hline & & C & 8,20 & 8,60 & 10,30 \\
\hline \multirow[t]{2}{*}{${ }^{16} \mathrm{O}(\mathrm{n}, \alpha){ }^{13} \mathrm{C}^{*}$} & $\mathrm{E}_{\boldsymbol{\gamma}} \sim 7,0 \mathrm{MeV}$ & $\alpha$ & 12,10 & 12,10 & 12,10 \\
\hline & & C & 12,10 & 12,10 & 13,30 \\
\hline
\end{tabular}

E* - Energie d'excitation

$E_{\gamma}-$ Energie des photons $\gamma$ émis

$Q$ - Energie de la réaction 
les valeurs des sections efficaces sont extraites des travaux cités par AUXIER et al. [12] et certaines des travaux [13-16].

Les valeurs des $\eta_{i j}\left(E_{n}\right)$ dépendent des critères de développabilité d'une trace. En général, celle-ci est fonction du pouvoir d'arrêt du matériau pour la particule considérée [1], on considère aussi que les traces des particules dont le parcours est inférieur à 1 ou $2 \mu \mathrm{m}$ ne sont pas décelables [17]*. Ces deux grandeurs dépendent de l'énergie de la particule, c'est pourquoi nous exprimons la développabilité d'une trace au moyen de l'énergie minimale de la particule. Nous considérons séparément les particules alpha et les noyaux de recul (c'est-à-dire $\mathrm{Be}, \mathrm{B}, \mathrm{C}, \mathrm{N}$ et $\mathrm{O}$ ), les énergies minimales supposées sont regroupées pour former trois groupes de restrictions énergétiques. Les groupes sont présentés dans le tableau II. Pour le calcul des $\eta_{i j}\left(E_{n}\right)$ nous avons fait les hypothèses suivantes :

- Nous considérons que la diffusion élastique est isotrope dans le système du centre de masse. Les valeurs sont obtenues à partir des valeurs de l'angle solide dans lequel sont éjectés les noyaux de recul dont l'énergie est supérieure aux énergies minimales mentionnées dans le tableau II. L'énergie d'un noyau de recul est calculée en utilisant les lois de conservation de l'énergie cinétique et de la quantité de mouvement. A l'aide des mêmes équations on détermine l'énergie minimale du neutron pour laquelle l'énergie des noyaux de recul dépasse le seuil mentionné dans le tableau II. Ces énergies minimales des neutrons sont données dans le tableau I pour les trois groupes de restrictions énergétiques.

- Quant aux autres réactions, nous supposons que les particules chargées lourdes ont une trace développable si leur énergie moyenne est supérieure aux seuils mentionnés dans le tableau II. A partir des mêmes lois que précédemment nous calculons l'énergie minimale des neutrons nécessaire pour qu'une particule produite forme une trace développable. Elles sont mentionnées dans le tableau I. Dans quelques cas, une réaction donne plusieurs particules chargées lourdes ; les facteurs $\eta_{i j}\left(E_{n}\right)$ peuvent prendre alors des valeurs supérieures à 1 .

TABLEAU II

GROUPES DE RESTRICTIONS ENERGETIQUES

\begin{tabular}{|c|c|c|}
\hline Groupe & Particule & $\begin{array}{c}\text { Particule enregistrée } \\
\text { si son énergie est supérieure à }\end{array}$ \\
\hline \multirow{2}{*}{ I } & $\begin{array}{c}\text { alpha } \\
\text { noyaux de recul }\end{array}$ & $0,2 \mathrm{MeV}$ \\
\hline \multirow{2}{*}{ II } & $\begin{array}{c}\text { alpha } \\
\text { noyaux de recul }\end{array}$ & $\begin{array}{c}0,2 \mathrm{MeV} \\
0,6 \mathrm{MeV}\end{array}$ \\
\hline \multirow{2}{*}{ III } & $\begin{array}{c}\text { alpha } \\
\text { noyaux de recul }\end{array}$ & $0,5 \mathrm{MeV}$ \\
$1,0 \mathrm{MeV}$
\end{tabular}

(*) La développabilité dépend aussi de l'angle entre la trajectoire de la particule et la surface du détecteur. On ne tient pas compte de cette influence dans le calcul. 
La développabilité et l'enregistrement des traces de plusieurs particules provenant de la même réaction est un problème délicat *. C'est pourquoi nous avons admis deux approximations limitatives. Dans la première (approximation A) on suppose que toutes les particules émises lors d'une réaction et dont l'énergie moyenne dépasse les seuils exigés sont enregistrables ; dans la deuxième $(B)$ on suppose qu'une seule particule chargée lourde est enregistrable. Le seuil d'enregistrement correspond dans ce dernier cas au seuil le plus bas, c'est-à-dire celui correspondant à la particule lourde formée la moins massive.

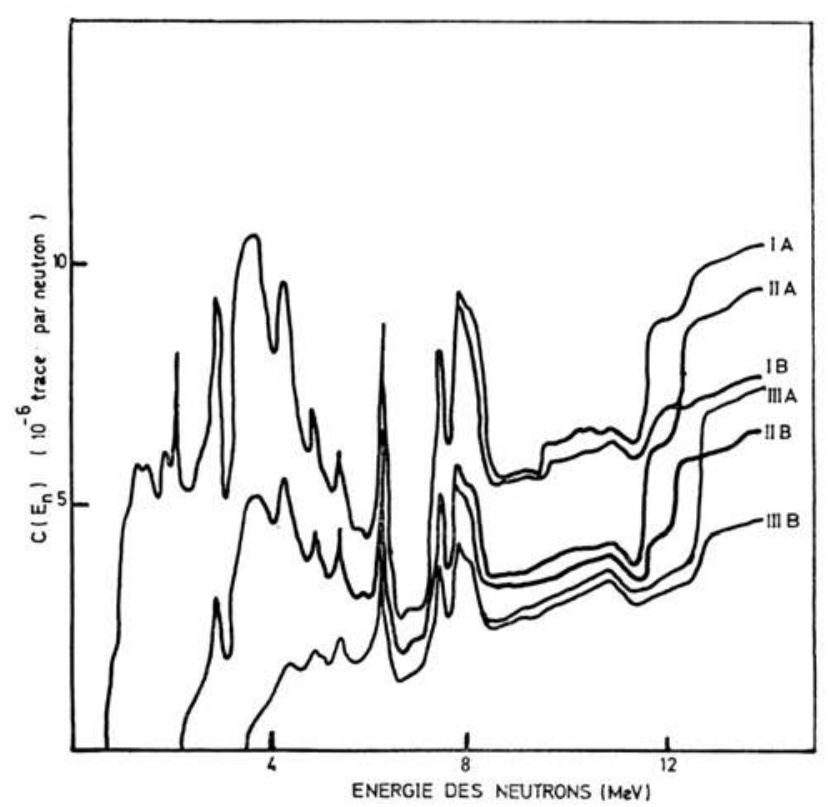

FIG. 1. - Valeurs des $C\left(E_{n}\right)$ pour le polycarbonate suivant les hypothèses utilisées A et B et les groupes de restrictions énergétiques.

Tant les valeurs des $\sigma_{i j}\left(E_{n}\right)$ que des $\eta_{i j}\left(E_{n}\right)$ étaient trouvées et/ou calculées pour chaque $E_{n}+\Delta E_{n}$, où $\Delta E_{n}=0,1 \mathrm{MeV}$ jusqu'à $E_{n}=14 \mathrm{MeV}$; dans les régions où $\sigma_{i j}\left(E_{n}\right)$ n'est pas donné de façon continue, les valeurs intermédiaires sont obtenues par interpolation. Les valeurs des $C\left(E_{n}\right)$ sont calculées pour le polycarbonate et les composés cellulosiques dont les compositions sont données dans le tableau III. Nous avons pris pour toutes les réactions et les particules produites $\Delta X=1 \mu \mathrm{m}$, les valeurs de $C\left(E_{n}\right)$ pour le polycarbonate et l'acétobutyrate de cellulose sont présentées dans les figures 1,2 . On y voit que les valeurs de $C\left(E_{n}\right)$ pour les deux matériaux sont de l'ordre de $10^{-6}$

(*) Par exemple, BENTON [18] a étudié l'efficacité d'enregistrement des trois particules émises lors de la réaction ${ }^{12} \mathrm{C}\left(n, n^{\prime}\right) 3 \alpha$; on pourrait déduire de ses résultats que cette efficacité est $\approx 10 \%$. vOL. $9-\mathrm{N}^{\circ} 4$ 
trace par neutron, les dépendances énergétiques sont semblables. (Les valeurs $C\left(E_{n}\right)$ obtenues pour le nitrate et le triacétate de cellulose ont une variation

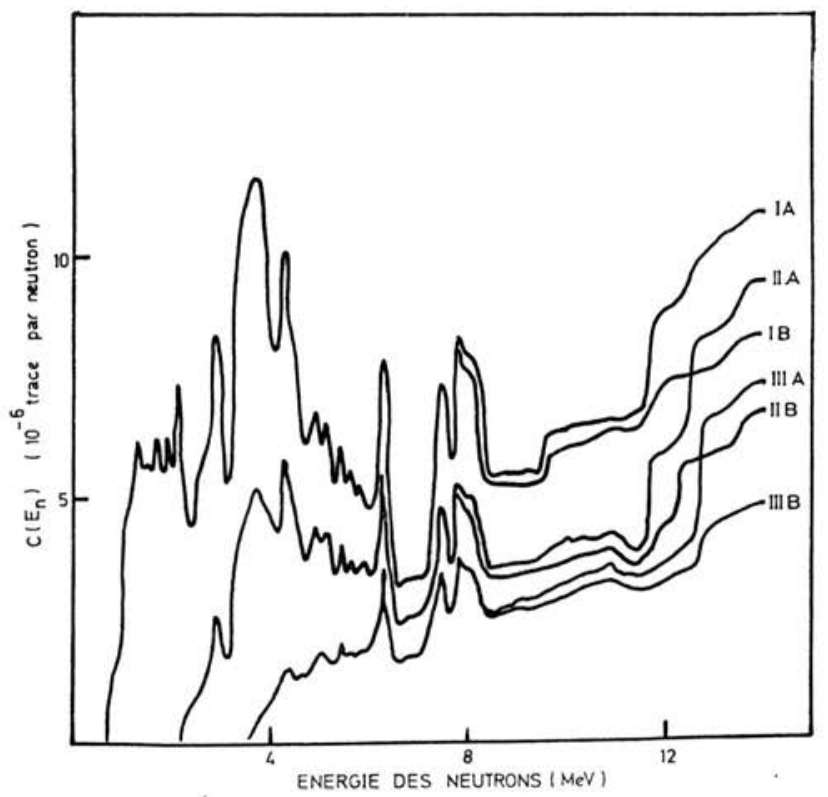

FIG. 2. - Valeurs des $C\left(E_{n}\right)$ pour l'acétobutyrate de cellulose suivant les hypothèses utilisées A et B et les groupes de restrictions énergétiques.

\section{TABLEAU III}

TENEUR EN CARBONE, AZOTE ET OXYGENE DES MATERIAUX PLASTIQUES ÉTUDIES

\begin{tabular}{|c|c|c|}
\hline Matériau & Noyau & $\begin{array}{c}\mathrm{N}_{\mathrm{j}} \\
\mathrm{cm}^{-3}\end{array}$ \\
\hline polycarbonate & $\mathrm{C}$ & $4,55.10^{22}$ \\
& $\mathrm{O}$ & $0,85.10^{22}$ \\
\hline acétobutyrate & $\mathrm{C}$ & $3,50.10^{22}$ \\
& $\mathrm{O}$ & $1,95.10^{22}$ \\
\hline triacétate de cellulose & $\mathrm{C}$ & $3,23.10^{22}$ \\
& $\mathrm{O}$ & $2,09.10^{22}$ \\
\hline nitrate de cellulose & $\mathrm{C}$ & $1,91.10^{22}$ \\
& $\mathrm{~N}$ & $0,77.10^{22}$ \\
& $\mathrm{O}$ & $3,45.10^{22}$ \\
\hline
\end{tabular}


très semblable à celle présentée dans les figures 1 et 2 [19]). Il s'ensuit que le choix de l'approximation A ou B est peu important pour les énergies $E_{n}<11 \mathrm{MeV}$; au-delà la différence est plus sensible surtout à cause de la réaction ${ }^{12} \mathrm{C}\left(\mathrm{n}, \mathrm{n}^{\prime}\right) 3 \alpha$. Ce fait général est dû à la prédominance des noyaux de recul provenant des diffusions élastiques dans le calcul de $C\left(E_{n}\right)$. La participation des particules alpha est alors assez faible (voir les figures 3 et 4 ).

Dans les cas pratiques on rencontre le plus souvent des neutrons polyénergétiques. Soit $\varphi\left(E_{n}\right)$ la fluence différentielle des neutrons

$$
\int_{E_{n}} \varphi\left(E_{n}\right) \mathrm{d} E_{n}=1 .
$$

Le nombre de particules produites par 1 neutron de cette source dans la couche du matériau $\Delta X=1 \mu \mathrm{m}$ et dont les traces sont développables est

$$
C(\text { source })_{\text {théor }}=\int_{E_{n}} C\left(E_{n}\right) \varphi\left(E_{n}\right) \mathrm{d} E_{n} .
$$

Nous avons calculé cette valeur pour les neutrons des sources Am-F et $\mathrm{Am}-\mathrm{Be}$, les neutrons rapides de fission et les neutrons de $14 \mathrm{MeV}$. Les spectres des sources Am-F et Am-Be sont ceux donnés par BURGER et al. [20]. Pour les neutrons de fission on a utilisé le spectre de WATT [21]. Les valeurs

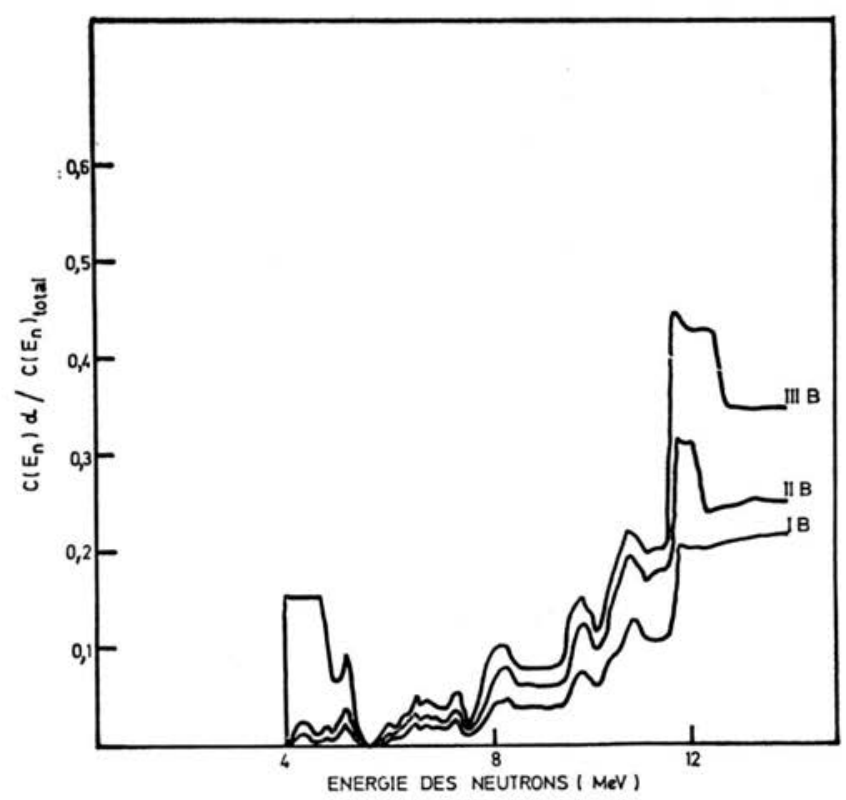

FIG. 3. - Taux de participation des particules alpha dans $C\left(E_{n}\right)$ pour le polycarbonate. 
des C (source) obtenues sont présentées dans le tableau IV. Les résultats sont donnés pour l'approximation B, probablement plus proche de la réalité. Les valeurs pour l'approximation A sont identiques sauf pour les neutrons de $14 \mathrm{MeV}$ où elles sont plus grandes d'environ $27 \mathrm{p}$. cent.

Dragu et Nicolae ont récemment calculé $\mathrm{C}(\mathrm{PuBe})$ pour le nitrate de cellulose [11]. Avec des hypothèses différentes des nôtres et plus simplifiées, ils ont obtenu la valeur de $5,46.10^{-6}$ trace par neutron (pour $\Delta X=1 \mu \mathrm{m}$ ), dont $4,0.10^{-7}$ trace par neutron pour les particules alpha. Notre valeur pour les particules alpha dépend peu du groupe de restriction énergétique; elle est égale, pour le nitrate de cellulose et les neutrons de la source Am-Be, à $3.10^{-7}$ trace par neutron, c'est-à-dire comparable à la leur. Les hypothèses utilisées dans le travail cité pour la diffusion élastique sont trop simplifiées pour qu'on puisse comparer les autres résultats.

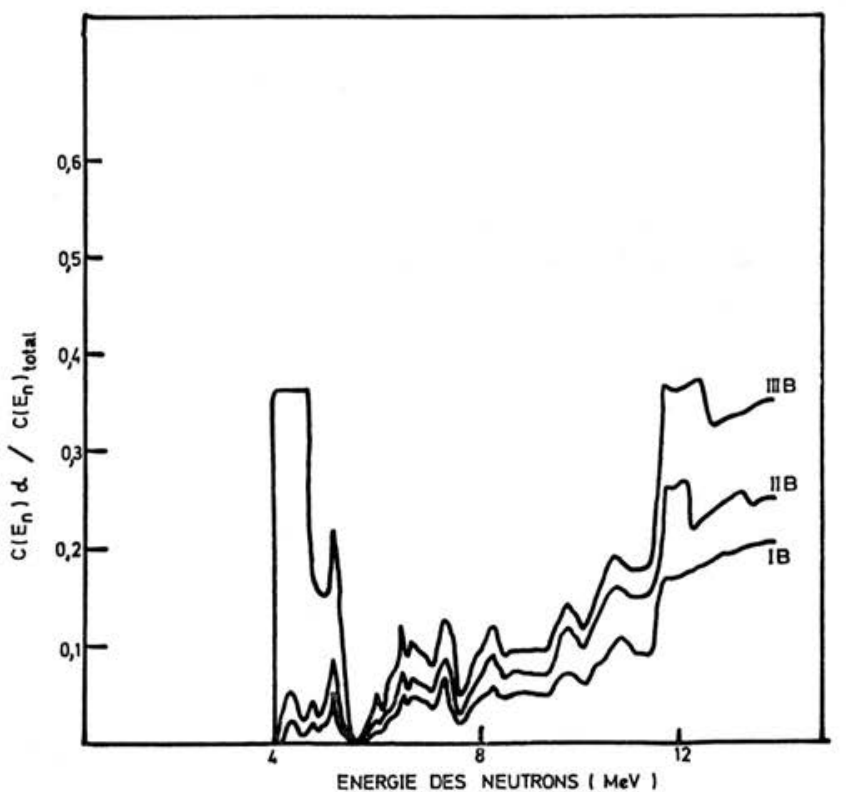

FIG. 4. - Taux de participation des particules alpha dans $C\left(E_{n}\right)$ pour l'acétobutyrate de cellulose.

\section{RÉSULTATS EXPÉRIMENTAUX}

Nous avons étudié l'enregistrement des neutrons dans les matériaux plastiques suivants :

- le triacétate de cellulose Triafol TN (Bayer, RFA), épaisseur $100 \mu \mathrm{m}$,

- le polycarbonate MAKRoFol E (Bayer, RFA) épaisseur $200 \mu \mathrm{m}$, 
TABLEAU IV

VALEURS DES C(source) théor POUR $\triangle \mathrm{V}=1 \mu \mathrm{m}$

ET LES MATERIAUX PLASTIQUES ETUDIES

\begin{tabular}{|c|c|c|c|c|c|}
\hline \multirow{2}{*}{ Source } & \multirow{2}{*}{$\begin{array}{l}\text { Groupe des } \\
\text { restrictions } \\
\text { énergétiques }\end{array}$} & \multicolumn{4}{|c|}{ Valeurs des $\mathrm{C}$ (source) théor, en trace par neutron } \\
\hline & & polycarbonate & $\begin{array}{l}\text { acétobutyrate } \\
\text { de cellulose }\end{array}$ & $\begin{array}{l}\text { triacétate } \\
\text { de cellulose }\end{array}$ & $\begin{array}{c}\text { nitrate } \\
\text { de cellulose }\end{array}$ \\
\hline Am - F & $\begin{array}{l}\text { I } \\
\text { II } \\
\text { III }\end{array}$ & $\begin{array}{l}4,65 \cdot 10^{-6} \\
1,49 \cdot 10^{-7} \\
3,95 \cdot 10^{-9}\end{array}$ & $\begin{array}{l}4,56 \cdot 10^{-6} \\
1,24 \cdot 10^{-7} \\
3,25 \cdot 10^{-9}\end{array}$ & $\begin{array}{l}4,41 \cdot 10^{-6} \\
1,20 \cdot 10^{-7} \\
3,09 \cdot 10^{-9}\end{array}$ & $\begin{array}{l}4,78 \cdot 10^{-6} \\
1,21 \cdot 10^{-7} \\
2,81 \cdot 10^{-8}\end{array}$ \\
\hline $\begin{array}{c}\text { neutrons } \\
\text { de } \\
\text { fission }\end{array}$ & $\begin{array}{c}\text { I } \\
\text { II } \\
\text { III }\end{array}$ & $\begin{array}{l}4,78 \cdot 10^{-6} \\
1,12 \cdot 10^{-6} \\
2,38 \cdot 10^{-7}\end{array}$ & $\begin{array}{l}4,72 \cdot 10^{-6} \\
1,04 \cdot 10^{-6} \\
2,16 \cdot 10^{-7}\end{array}$ & $\begin{array}{l}4,49.10^{-6} \\
1,00.10^{-6} \\
2,08 \cdot 10^{-7}\end{array}$ & $\begin{array}{l}5,09.10^{-6} \\
1,09.10^{-6} \\
2,64.10^{-7}\end{array}$ \\
\hline $\mathrm{Am}-\mathrm{Be}$ & $\begin{array}{l}\text { I } \\
\text { II } \\
\text { III }\end{array}$ & $\begin{array}{l}6,59 \cdot 10^{-6} \\
3,34 \cdot 10^{-6} \\
1,33 \cdot 10^{-6}\end{array}$ & $\begin{array}{l}6,61 \cdot 10^{-6} \\
3,24 \cdot 10^{-6} \\
1,24 \cdot 10^{-6}\end{array}$ & $\begin{array}{l}6,30.10^{-6} \\
3,09.10^{-6} \\
1,18.10^{-6}\end{array}$ & $\begin{array}{l}7,41 \cdot 10^{-6} \\
3,54 \cdot 10^{-6} \\
1,37 \cdot 10^{-6}\end{array}$ \\
\hline $14 \mathrm{MeV}$ & $\begin{array}{l}\text { I } \\
\text { II } \\
\text { III }\end{array}$ & $\begin{array}{l}7,70 \cdot 10^{-6} \\
6,57 \cdot 10^{-6} \\
4,73 \cdot 10^{-6}\end{array}$ & $\begin{array}{l}8,44 \cdot 10^{-6} \\
6,80 \cdot 10^{-6} \\
4,89 \cdot 10^{-6}\end{array}$ & $\begin{array}{l}8,19 \cdot 10^{-6} \\
6,51 \cdot 10^{-6} \\
4,80.10^{-6}\end{array}$ & $\begin{array}{r}10,20.10^{-6} \\
7,65 \cdot 10^{-6} \\
5,58 \cdot 10^{-6}\end{array}$ \\
\hline
\end{tabular}

- l'acétobutyrate de cellulose (Synthesia Semtin, Tchécoslovaquie), épaisseur $180 \mu \mathrm{m}$.

Les films des matériaux sont irradiés par les neutrons des sources Am-F, $\mathrm{Am}-\mathrm{Be}$ et par les neutrons de $14 \mathrm{MeV}$ sous une incidence normale *. Les fluences de neutrons des sources $\mathrm{Am}-\mathrm{F}$ et $\mathrm{Am}-\mathrm{Be}$ sont déterminées à partir des émissions totales, la correction pour non-ponctualité étant calculée d'après [22]. Les fluences de neutrons de $14 \mathrm{MeV}$ sont mesurées au moyen d'un détecteur à activation basé sur la réaction ${ }^{63} \mathrm{Cu}(\mathrm{n}, 2 \mathrm{n})^{62} \mathrm{Cu}$. Les fluences appliquées sont, pour toutes les sources, de 2 à $3.10^{9} \mathrm{n} . \mathrm{cm}^{-2}$; on irradie toujours des «sandwiches » constitués de 5 films, les trois feuilles internes étant seules prises en compte pour la mesure.

Les matériaux sont ensuite soumis à différentes attaques chimiques, soit par la soude en solution aqueuse $(6,8$ et $10 \mathrm{~N})$ soit par les solutions recommandées par SOMOGYI [23], c'est-à-dire et

$20 \mathrm{~g} \mathrm{NaOH}+16 \mathrm{~g} \mathrm{KOH}_{+4,5} \mathrm{~g} \mathrm{KMnO}_{4}+100 \mathrm{~g} \mathrm{H}_{2} \mathrm{O}$ (solution « $\mathrm{B}$ »)

$15 \mathrm{~g} \mathrm{KOH}+40 \mathrm{~g} \mathrm{C}_{2} \mathrm{H}_{5} \mathrm{OH}+45 \mathrm{~g} \mathrm{H}_{2} \mathrm{O}$ (solution « $\mathrm{S} »$ ).

(*) Cela permet d'éviter ou tout au moins de minimiser l'influence de l'angle critique $\theta_{c}$ [1] sur l'efficacité d'enregistrement et cela permet donc de comparer valablement les résultats expérimentaux avec le calcul théorique.

vol. $9-\mathrm{N}^{\circ} 4$ 
Les températures d'attaque sont 50 et/ou $60^{\circ} \mathrm{C}$ pour les solutions de soude, $70^{\circ} \mathrm{C}$ dans le cas des solutions « $\mathrm{B}$ » et « $\mathrm{S}$ ». Les épaisseurs des films avant et après attaque sont déterminées au moyen d'un optimètre Zeiss et à l'aide de la pesée.

Les traces sont comptées à l'aide d'un microscope optique Zeiss. Toutes les lectures sont effectuées avec le grossissement $630 \times$; nous comptons toutes les traces dont la plus petite dimension est supérieure à $1,8 \mu \mathrm{m}$. Le bruit de fond, c'est-à-dire le comptage dans le matériau non irradié est évalué dans les mêmes conditions.

Le nombre de traces comptées ne dépend pas seulement des paramètres physiques mentionnés dans la partie théorique. Il dépend aussi des caractéristiques de la méthode d'attaque chimique, tout d'abord des paramètres qui influencent le rapport $V_{t} / V_{m} \quad\left(V_{t}=\right.$ vitesse d'attaque le long de la trace et $V_{m}=$ vitesse d'attaque en masse du détecteur) [1,24]. De plus, il dépend de la durée de la décelabilité d'une trace au cours d'une attaque continue. Nous avons montré dans un travail récent [25] que, de ce point de vue, l'attaque par les solutions de SomogYI apporte un avantage assez important. Nous avons observé que dans ce cas on peut bien voir dans la même image les traces de
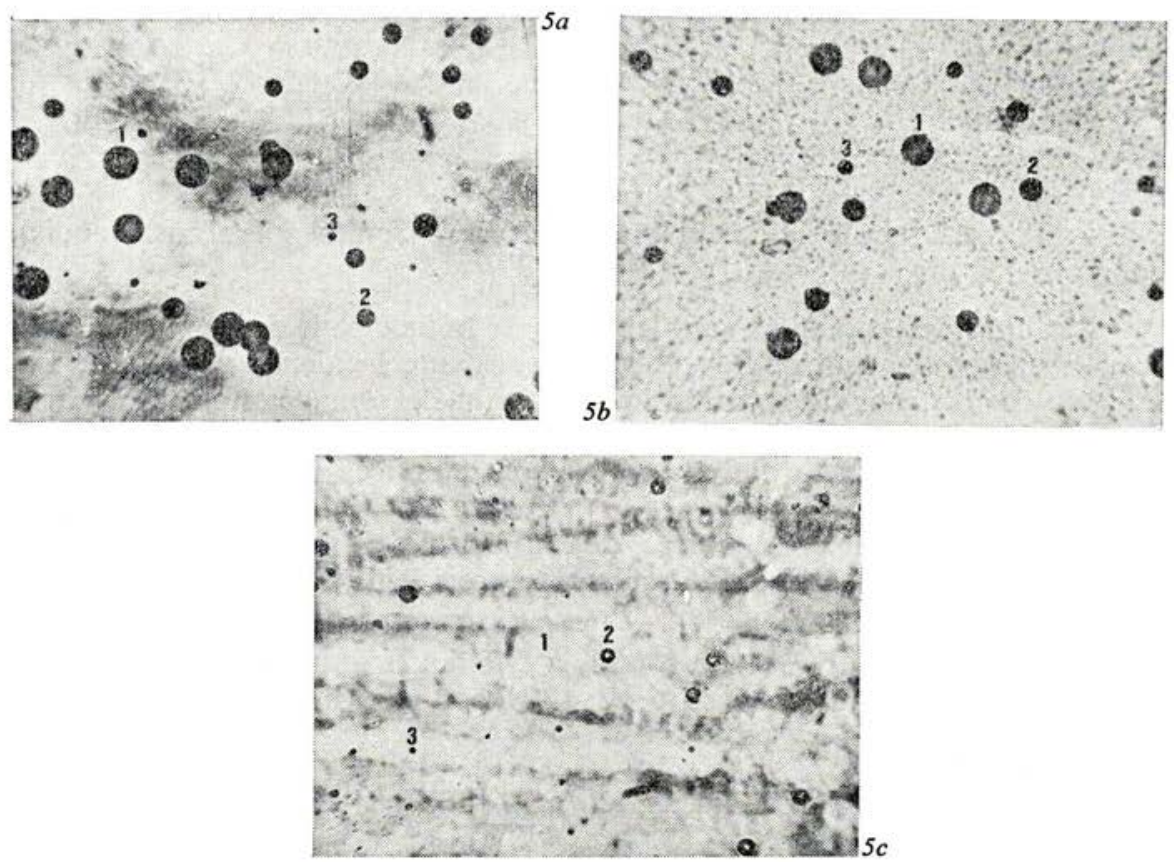

FIG. 5. - Microphotographies des traces de particules alpha de $1,5 \mathrm{MeV}$ (1), $3 \mathrm{MeV}$ (2), et 4,4 MeV (3) entrant perpendiculairement dans le matériau. $a$. MAKROFOL E, attaqué par la solution " $S$ " à $70^{\circ} \mathrm{C}, h \approx 18 \mu \mathrm{m} ; b$. Acétobutyrate de cellulose, attaqué par la solution « $B$ » à $70^{\circ} \mathrm{C}, h \approx 20 \mu \mathrm{m} ; c$. Triafol TN, attaqué par la soude $(10 \mathrm{~N})$ en solution aqueuse à $60^{\circ} \mathrm{C}, h \approx 10 \mu \mathrm{m}$. 
particules alpha d'énergies très différentes, ce qui est plus difficile dans le cas de l'attaque par la soude (voir la figure 5), l'attaque par les solutions « B » et « $\mathrm{S}$ » est, de plus, bien plus rapide $[23,25]$. Si nous revenons au cas des neutrons rapides, on peut s'attendre à ce que l'influence de cette durée de décelabilité soit encore plus importante car les particules sont produites à l'intérieur même du détecteur.

Effectivement, nous avons observé que les densités de traces après attaque par la soude en solution aqueuse sont nettement inférieures à celles obtenues avec les solutions « $\mathrm{B}$ » et « S ». A titre d'exemple, la sensibilité pour l'acétobutyrate et le triacétate de cellulose attaqué par la soude $(6$ et/ou $10 \mathrm{~N}$ en solution aqueuse à $50 \mathrm{et} / \mathrm{ou} 60^{\circ} \mathrm{C}$ ) n'est au maximum que de $5.10^{-6}$ trace par neutron pour les neutrons de la source Am-Be. C'est pourquoi nous avons étudié la dépendance énergétique surtout dans les cas de l'attaque par les solution « $\mathrm{S}$ » (polycarbonate MAKROFOL) et « B » (acétobutyrate de cellulose). Les résultats obtenus sont présentés dans les figures 6 et 7 .

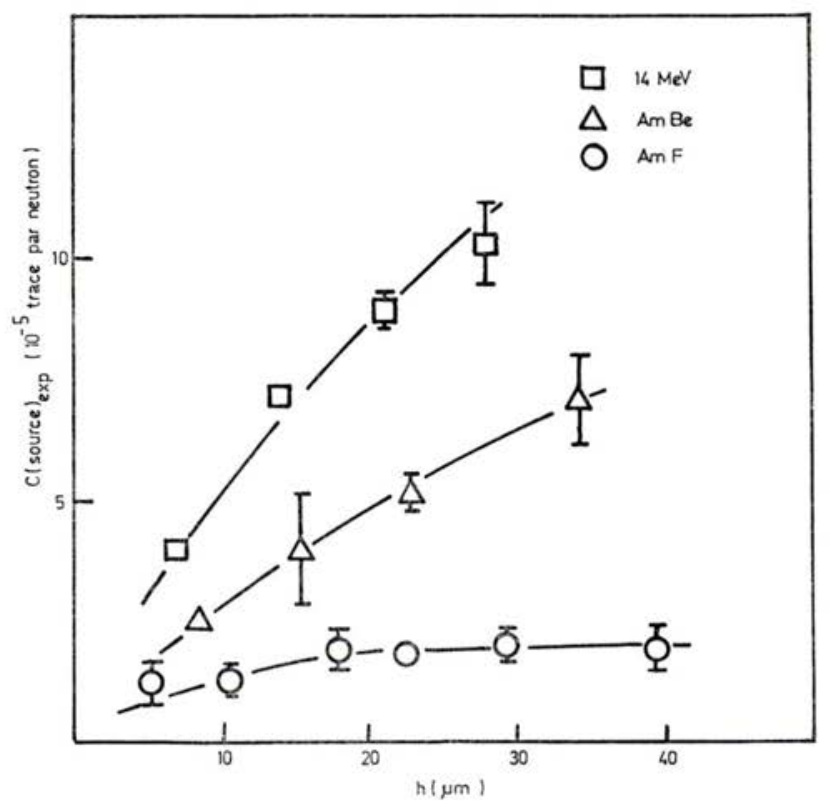

FIG. 6. - Variation de $C$ (source) exp en fonction de l'épaisseur attaquée $h$; MAKRofol E attaqué par la solution $« S »$ à $70^{\circ} \mathrm{C}$.

On remarque que :

- les sensibilités diminuent dans l'ordre $14 \mathrm{MeV}>\mathrm{Am}-\mathrm{Be}>\mathrm{Am}-\mathrm{F}$, quelle que soit l'épaisseur $h$ détachée de la surface du matériau par attaque ; elles sont semblables dans les deux cas étudiés ;

- les sensibilités augmentent avec l'épaisseur $h$; cette augmentation 
dépend de l'énergie des neutrons et varie dans le même sens, c'est-à-dire qu'elle est plus importante dans le cas des neutrons de $14 \mathrm{MeV}$.

On peut constater que les sensibilités que nous avons déterminées sont généralement supérieures à celles publiées autrefois dans les cas d'attaque par la soude ou la potasse en solutions aqueuses $[4,6,7,10,11]$. D'après les remarques précédentes, ce fait n'est pas surprenant. Il faut remarquer que NishiwAKI et al. [6] ont observé un effet semblable dans le cas d'un polycarbonate. Ils ont trouvé pour la potasse en solution aqueuse

$$
C(14 \mathrm{MeV})_{\exp } \leqslant 2.10^{-5} \text { trace par neutron, }
$$

tandis que l'attaque par la potasse en solution d'alcool méthylique a donné, par exemple,

$$
C(14 \mathrm{MeV}) \approx 6,3 \cdot 10^{-5} \text { trace par neutron }
$$

pour l'épaisseur $h \approx 13 \mu \mathrm{m}$. On voit sur la figure 6 que notre valeur est très proche de la sienne. Dans un travail récent [26] KHAN a également observé une sensibilité assez élevée dans le cas du nitrate de cellulose attaqué par la potasse en solution aqueuse et les neutrons de $14 \mathrm{MeV}$.

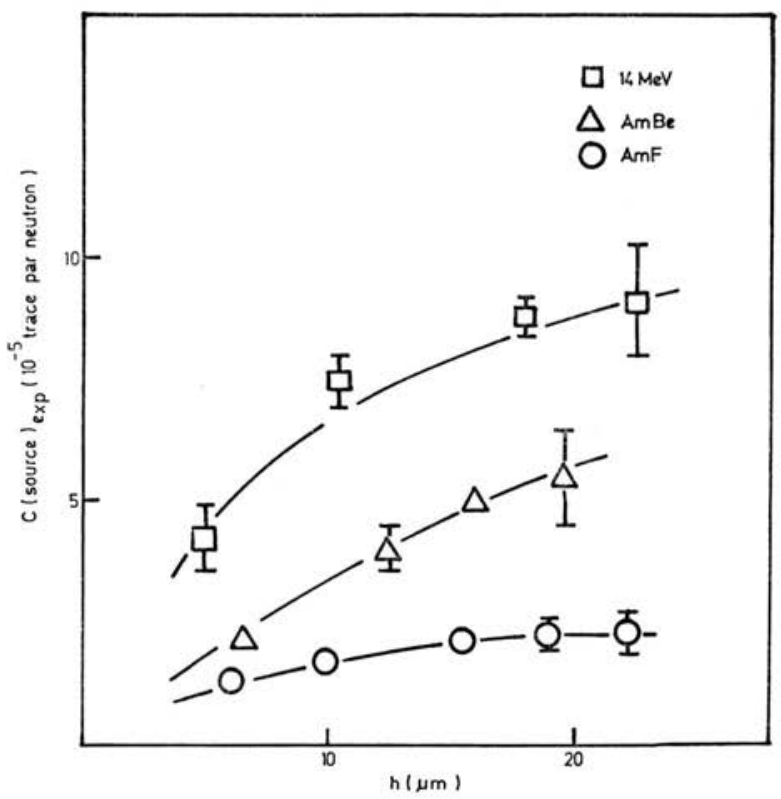

FIg. 7. - Variation de $C$ (source) exp en fonction de l'épaisseur attaquée $h$; acétobutyrate de cellulose attaqué par la solution $« B »$ à $70^{\circ} \mathrm{C}$.

\section{DISCUSSION}

Deux facteurs principaux interviennent dans les dépendances observées : le premier est fonction des paramètres physiques et est exprimé par les valeurs 


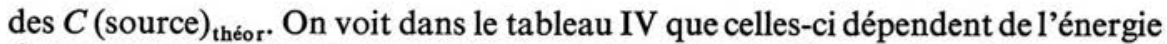
des neutrons de la même manière que les valeurs $C$ (source) exp. $_{\text {. }}$ Mais, avant de comparer les résultats expérimentaux et théoriques, il faut discuter de l'influence du deuxième facteur ayant trait aux conditions de l'attaque et leur influence sur le nombre de traces comptées.

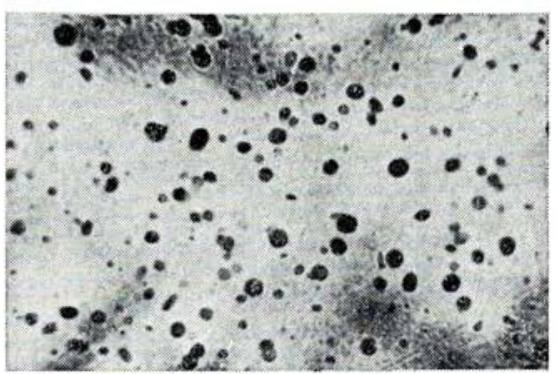

$8 a$

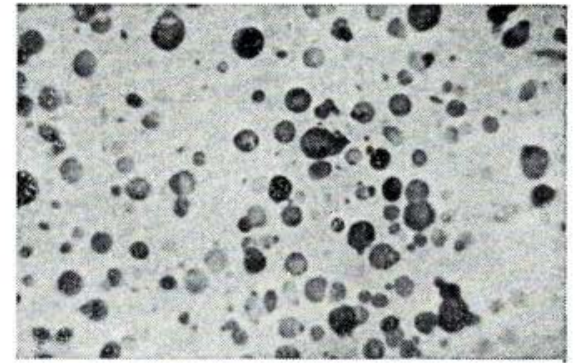

$8 b$

FIG. 8. - Microphotographies de traces de particules chargées lourdes produites dans le MAKrofol E par les neutrons de $14 \mathrm{MeV}$. $a$. attaqué $20 \mathrm{~min}$ par la solution " $S$ » à $70^{\circ} \mathrm{C}(h=7 \mu \mathrm{m}) ; b$. attaqué $40 \mathrm{~min}$ par la solution « $S$ » à $70^{\circ} \mathrm{C}(h=14 \mu \mathrm{m})$.

Quand l'attaque continue, l'épaisseur $h$ augmente ; on observe deux phénomènes : d'une part, les dimensions des traces déjà visibles grandissent, d'autre part, les traces des particules produites à l'intérieur du détecteur commencent à être développées. On peut donc s'attendre à ce que le nombre de traces ainsi que leur dimension moyenne croîssent avec l'épaisseur $h$ (voir la figure 8$)[2,6,7,26]$. Ce processus mène à un certain équilibre dans lequel les traces qui cessent d'être bien visibles sont remplacées par des traces nouvelles. En même temps, tant l'augmentation initiale du nombre des traces que le niveau de cet équilibre devrait dépendre de l'énergie des neutrons. Effectivement, l'énergie et le parcours moyen des particules chargées produites augmentent avec l'énergie des neutrons et nous avons observé [25] que la durée de la décelabilité d'une trace (exprimée par l'épaisseur $h$ ) augmente avec le parcours d'une particule. On peut donc s'attendre à ce que, d'une part, l'augmentation initiale du nombre de traces comptées soit plus significative pour les neutrons d'énergie élevée, d'autre part, l'équilibre mentionné plus haut soit obtenu plus tard pour les neutrons plus énergétiques, c'est-à-dire pour une épaisseur plus grande. On voit dans les figures 6 et 7 que ces dépendances sont effectivement observées *.

Revenons maintenant à la comparaison de nos résultats expérimentaux avec l'analyse théorique. Si l'équilibre mentionné ci-dessus est achevé, on peut écrire :

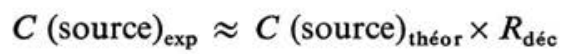

(*) Nous avons effectué quelques expériences pour $h>30 \mu \mathrm{m}$ dans les cas des neutrons Am-Be et $14 \mathrm{MeV}$. Les résultats obtenus sont assez peu reproductibles. L'évaluation se heurte à l'obstacle provenant du fait qu'il y a dans l'image beaucoup de traces «vieilles» et très grandes; elle devient très compliquée. 
où $R_{\mathrm{dés}}$ représente une couche maximale caractérisée en ce que les traces des particules sont bien décelables.

D'après les remarques précédentes $R_{\mathrm{déc}}$ devrait croître avec l'énergie

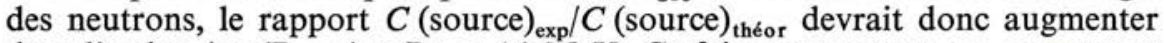
dans l'ordre Am-F $<\mathrm{Am}-\mathrm{Be}<14 \mathrm{MeV}$. Ce fait nous permet, en comparant les résultats expérimentaux et théoriques, d'apprécier quel est le groupe de restrictions énergétiques le plus valable, c'est-à-dire à partir de quelle énergie les particules créent des traces enregistrables dans nos conditions expérimentales (voir tableau II).

\section{TABLEAU V}

VALEURS DU RAPPORT $\frac{\text { C(source) exp. POUR L'EPAISSEUR } h \sim 20 \mu \mathrm{m}}{\text { C(source) théor }}$

ET LES TROIS GROUPES DE RESTRICTIONS ENERGÊTIQUES

\begin{tabular}{|l|c|r|r|r|}
\hline \multirow{2}{*}{ Matériau } & \multirow{2}{*}{ Source } & \multicolumn{3}{|c|}{ C(source $\exp$ / C(source) théor pour le groupe } \\
\cline { 3 - 5 } & & I & II & III \\
\cline { 3 - 5 } & \multirow{2}{*}{ Am-F } & 4,1 & 127,5 & 4810,0 \\
Polycarbonate & Am-Be & 7,3 & 14,4 & 36,1 \\
MAKROFOL E & $14 \mathrm{MeV}$ & 11,2 & 13,1 & 18,2 \\
& Am-F & 4,8 & 177,4 & 6769,2 \\
& Am-Be & 8,5 & 17,3 & 45,2 \\
Acétobutyrate & $14 \mathrm{MeV}$ & 10,7 & 13,2 & 18,4 \\
de cellulose & &
\end{tabular}

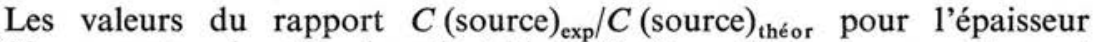
$h \approx 20 \mu \mathrm{m}$ sont présentées dans le tableau V. On voit que le comportement attendu n'est trouvé que pour le groupe de restrictions énergétiques I *. Ceci nous permet de supposer que les noyaux de recul autant que les particules alpha forment des traces développables dès $\approx 0,2 \mathrm{MeV}$. Cette constatation recoupe les résultats obtenus avec la source Am-F. En effet, le nombre de particules alpha créées par 1 neutron de la source Am-F est très faible (voir les figures 3 et 4 ). Les valeurs théoriques pour la couche

$$
\Delta X=1 \mu \mathrm{m}\left(E_{\alpha} \geqslant 0,2 \mathrm{MeV}\right)
$$

sont respectivement $8.10^{-11}$ et $1,8 \cdot 10^{-10}$ trace par neutron pour le polycarbonate et l'acétobutyrate de cellulose. Le rapport $C(\mathrm{Am}-\mathrm{F})_{\text {exp }} / C(\mathrm{Am}-\mathrm{F})_{\text {théor }}$ donne dans ce cas des valeurs de $R_{\text {déc }}$ de l'ordre de $10^{3}$ à $10^{5} \mu \mathrm{m}$, si seules les particules alpha sont enregistrées, valeurs qui sont inexplicables par rap-

(*) Evidemment, l'équilibre n'est pas obtenu pour les neutrons AmBe et les neutrons de $14 \mathrm{MeV}$. Mais on peut s'attendre à ce que les tendances observées soient semblables. 
port au parcours des particules alpha [28]. On doit donc admettre l'enregistrement des noyaux de recul d'énergie faible.

$R_{\mathrm{dec}}$ représente une épaisseur "utile " d'un matériau ; il est possible de le comparer avec le parcours moyen des particules chargées lourdes produites. Il faut d'abord souligner que les valeurs théoriques sont vraisemblablement un peu surestimées. Nous avons en effet supposé dans le calcul que la diffusion élastique est une réaction isotrope dans le système du centre de masse. Cette supposition n'est pas entièrement valable. Le calcul précis basé sur les données disponibles a montré que les valeurs exactes des $\eta_{i j}\left(E_{n}\right)$ sont, dans le cas du groupe de restrictions énergétiques I et pour $E_{n} \geqslant 3 \mathrm{MeV}$ environ $20 \mathrm{p}$. cent moins grandes [19]. Etant donné l'importance de la diffusion élastique dans

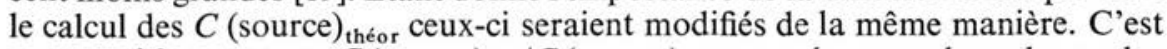
pourquoi les rapports $C$ (source) $)_{\text {exp }} / C$ (source) théor $_{\text {représentent des valeurs plus }}$ faibles que les épaisseurs utiles. Ceci est encore plus vrai dans le cas des neutrons $\mathrm{Am}-\mathrm{Be}$ et $14 \mathrm{MeV}$ où l'épaisseur $h \approx 20 \mu \mathrm{m}$ prise pour la comparaison n'est pas suffisante pour obtenir l'équilibre entre les traces nouvelles et celles qui disparaissent (voir les figures 6 et 7).

Les parcours moyens réels des particules chargées lourdes produites sont calculés à partir des données obtenues lors du calcul théorique et celles présentées dans le travail précédent [28] et ils sont égaux à environ 2,4 et $7 \mu \mathrm{m}$ pour les neutrons $\mathrm{Am}-\mathrm{F}, \mathrm{Am}-\mathrm{Be}$ et $14 \mathrm{MeV}$, respectivement. On voit d'après le tableau $\mathrm{V}$ que les valeurs $R_{\mathrm{déc}}$, bien qu'étant plus faibles que les valeurs des épaisseurs utiles sont nettement supérieures aux parcours moyens réels. Ceci provient du fait déjà signalé que la trace d'une particule est décelable, au cours d'une attaque continue, pendant un temps bien plus long que celui qui correspond à l'attaque d'une couche égale au parcours réel.

Les études que nous avons réalisées ne sont pas encore assez complètes pour que nous puissions envisager l'utilisation immédiate des détecteurs étudiés pour la dosimétrie. Néanmoins, nous pouvons estimer quelle serait la dépendance énergétique des détecteurs comme dosimètres de neutrons rapides. Si nous prenons comme facteurs de conversion $\mathrm{rad}^{*} / n . \mathrm{cm}^{2}$ ceux des travaux $[12,20]$, les densités de traces obtenues pour $h \approx 20 \mu \mathrm{m}$ correspondent aux sensibilités suivantes :

$5,6.10^{3} ; 9,7.10^{3}$ et $10,4 \cdot 10^{3}$ trace. $\mathrm{cm}^{-2} \cdot \mathrm{rad}^{-1}$ pour MAKROFOL $\mathrm{E}$ et $6,4 \cdot 10^{3} ; 11,4 \cdot 10^{3}$ et $10,8 \cdot 10^{3}$ trace. $\mathrm{cm}^{-2} \cdot \mathrm{rad}^{-1}$ pour l'acétobutyrate de cellulose, pour les neutrons $\mathrm{Am}-\mathrm{F}, \mathrm{Am}-\mathrm{Be}$ et $14 \mathrm{MeV}$, respectivement.

A partir de ces valeurs et des spectres neutroniques on peut apprécier que les détecteurs étudiés auraient une dépendance énergétique assez faible pour les neutrons dont l'énergie est supérieure à environ $1,3 \mathrm{MeV}$. Comme prévu théoriquement (voir les figures 1 et 2), la sensibilité aux neutrons d'énergie inférieure à cette valeur est faible.

(*) La dose de multicollisions dans le tissu.

voL. $9-\mathrm{N}^{\circ} 4$ 


\section{CONCLUSION}

Nos résultats principaux sont les suivants :

- nous avons étudié le nombre de traces créées par interaction des neutrons rapides avec les noyaux constituant des matériaux plastiques utilisés comme détecteurs solides de traces ;

- nous avons prouvé que ces traces sont décelables par une attaque chimique ; nous avons trouvé que la sensibilité de la méthode dépend de façon très importante des conditions de l'attaque chimique ;

- la comparaison des valeurs expérimentales et du calcul théorique a montré que les noyaux de recul ainsi que les particules alpha ont des traces décelables si leur énergie est au moins égale à $0,2 \mathrm{MeV} ; 1$ 'épaisseur utile d'un matériau dépend de l'énergie des neutrons, elle est nettement plus grande que le parcours moyen des particules chargées lourdes produites ;

- quant à l'application dosimétrique, les détecteurs étudiés devraient avoir une dépendance énergétique assez faible pour les neutrons dont l'énergie est comprise entre $1,3 \mathrm{MeV}$ et $14 \mathrm{MeV}$ environ ; la sensibilité dans cette région serait, pour nos conditions expérimentales, de l'ordre de :

$10^{4}$ trace. $\mathrm{cm}^{-2} \cdot \mathrm{rad}^{-1}$ pour une épaisseur $h$ détachée de la surface du matériau égale à $20 \mu \mathrm{m}$.

\section{REMERCIEMENTS}

Nous tenons à remercier Madame SimunKova pour son assistance dans l'évaluation des détecteurs.

Nous voudrions exprimer nos remerciements très vifs à Madame CHAPUIS pour ses conseils et son aide dans la rédaction finale de ce travail.

\section{BIBLIOGRAPHIE}

[1] BLANC D. - Radioprotection, 1970, 5, 37, 115, 201.

[2] BECKER K. - «Topics in radiation dosimetry ", suppl. n 1 (ATTIX F.H. et al., Eds). Academic press, New York, 1972, p. 79.

[3] Pretre S. - «Neutron monitoring for radiation protection purposes » IAEA, Vienna, 1973, vol. II, p. 99.

[4] BECKER K. - Health Physics, 1969, 16, 113.

[5] TUYN J.W.N. - Radiat. Effects, 1970, 5, 75.

[6] Nishiwaki Y., Tsuruba T., - J. Nucl. Sci. Technol., 1971, 8, 162.

[7] PIESCH (E.). - « Advances in physical and biological radiation detectors » IAEA, Vienna, 1971, p. 399.

[8] Sohrabi M., Becker K. - ORNL-TM-3605, 1971.

[9] TYMmons B.J., TUYN J.W.N., BAARLI J. - « Neutron monitoring for radiation protection purposes ", IAEA, Vienna, 1973, vol. II, p. 69.

[10] Jozefowicz K. - " Neutron monitoring for radiation protection purposes » IAEA. Vienna, vol. II, p. 183.

[11] Dragu A., Nicolae M. - Radioprotection, 1972, 7, 87. 
[12] Auxier J.A., SNyder W.S., Jones T.R. - « Radiation dosimetry » (AtTIX F.H. et al., Eds). Academic press, New York, 1972, vol. I, p. 275.

[13] BaCh R.L., Caswell P.S. - Radiat. Res., 1968, 35, 1.

[14] Hughes D.J., Schartz R.B. - " Neutron cross sections ", BNL-325, 2nd edition, 1958.

[15] Stehn J.R., Golberg M.D., Magurno S.A., Wienner-Chassman R. - " Neutron cross sections ", BNL-325, suppl. n² 2, 2nd edition, vol. I, 1964.

[16] Goldberg M.D., MAY V.M., STEHN J.R. - «Angular distributions in neutron induced reactions ", BNL-400, 2nd edition, vol. I, 1962.

[17] Fleisher R.L., Price P.B., Walker R.M., Hubbard E.L., - Phys. Rev., 1964, 133, 517.

[18] Frank A.L., Benton E.V. - Radiat. Effects, 1970, 3, 33.

[19] SPURNY F., Lochmanova J. - Jaderna Energie (à paraitre).

[20] Burger G., ECKL G., Gredel H. - « Advances in physical and biological radiation detectors, IAEA, Vienne, 1971, p. 467.

[21] Dahl R.E., Yoshikawa H.I. - « Neutron fluence measurements », IAEA, Vienna, 1970 , p. 141.

[22] Gusev N. et al. - " Zashchita of izlucheniya protiyazhennych istochnikov " (en russe), Gosatomizdat, Moscou, 1961.

[23] Sомоgyi G., Gulyas J. - Radioisotopy, 1972, 13, 549.

[24] Somogyi G., Szalay S.A. - Nucl. Instr. Meth., 1973, 109, 211.

[25] SPURNY F. - « Etudes des caractéristiques de certains matériaux plastiques comme les détecteurs solides de traces " (en tchèque). Rapport LRD CSAV 3/73, Prague, 1973.

[26] Khan H.A. - Nucl. Instr. Meth., 1973, 113, 55.

[27] Frank A.L., Benton E.V. - " C.R. $7^{\circ}$ colloque international de photographie corpusculaire et des détecteurs visuels solides ", Barcelona, 1970, p. 441.

[28] SPURNY F. - « Proc. 8 th international conference on nuclear photography and solid state track detectors ", Bucharest, 1972, vol. II, p. 355. 